City University of New York (CUNY)

CUNY Academic Works

\title{
Accuracy of highly sexually active gay and bisexual men's predictions of their daily likelihood of anal sex and its relevance for intermittent event-driven HIV Pre-Exposure Prophylaxis
}

Jeffrey T. Parsons

CUNY Hunter College

H. Jonathon Rendina

Center for HIV/AIDS Educational Studies and Training

Christian Grov

CUNY School of Public Health

Ana Ventuneac

Center for HIV/AIDS Educational Studies and Training

Brian Mustanski

Northwestern University

\section{How does access to this work benefit you? Let us know!}

More information about this work at: https://academicworks.cuny.edu/sph_pubs/62

Discover additional works at: https://academicworks.cuny.edu

This work is made publicly available by the City University of New York (CUNY).

Contact: AcademicWorks@cuny.edu 
Running Head: Predicting anal sex and its relevance for PrEP

Accuracy of highly sexually active gay and bisexual men's predictions of their daily likelihood of anal sex and its relevance for intermittent event-driven HIV Pre-Exposure Prophylaxis

Jeffrey T. Parsons, PhD, ${ }^{1,2,3,4}$ H. Jonathon Rendina, PhD, MPH, ${ }^{2}$ Christian Grov, $\mathrm{PhD}, \mathrm{MPH},{ }^{2,4,5}$ Ana Ventuneac, $\mathrm{PhD}^{2}$ \& Brian Mustanski ${ }^{6}$

${ }^{1}$ Department of Psychology, Hunter College of the City University of New York (CUNY), New York, NY, USA

${ }^{2}$ The Center for HIV/AIDS Educational Studies \& Training (CHEST), New York, NY, USA

${ }^{3}$ Health Psychology and Clinical Sciences Doctoral Program, The Graduate Center of the City University of New York (CUNY), New York, NY, USA

${ }^{4}$ CUNY School of Public Health, New York, NY, USA

${ }^{5}$ Department of Health and Nutrition Sciences, Brooklyn College of the City University of New York (CUNY), Brooklyn, NY, USA

${ }^{6}$ Department of Medical Social Sciences, Northwestern University Feinberg School of Medicine, Chicago, IL, USA 
Correspondence concerning this article should be addressed to Jeffrey T. Parsons, Department of Psychology, Hunter College of the City University of New York, 695 Park Ave., New York, NY 10065. Fax: 212-206-7994. Email: Jeffrey.Parsons@ @unter.cuny.edu

Conflicts of Interest and Sources of Funding: This project was supported by a research grant from the National Institute of Mental Health (R01-MH087714; Jeffrey T. Parsons, Principal Investigator). H. Jonathon Rendina was supported in part by a National Institute of Mental Health Ruth L. Kirchstein Individual Predoctoral Fellowship (F31-MH095622). No other conflicts are declared.

Portions of this manuscript have been previously presented at the $8^{\text {th }}$ International Conference on HIV Treatment and Prevention Adherence of the International Association of Providers in AIDS CARE (IAPAC) in Miami, Florida on June 4, 2013.

\begin{abstract}
Objective: We sought to examine highly sexually active gay and bisexual men's accuracy in predicting their sexual behavior for the purposes of informing future research on intermittent, event-driven HIV Pre-Exposure Prophylaxis (PrEP).

Design: For 30 days, 92 HIV-negative men completed a daily survey about their sexual behavior ( $n=1,688$ days of data) and indicated their likelihood of having anal sex with a casual male partner the following day.
\end{abstract}

Method: We utilized multilevel modeling to analyze the association between self-reported likelihood of and subsequent engagement in anal sex. 
Results: We found a linear association between men's reported likelihood of anal sex with casual partners and the actual probability of engaging in sex, though men overestimated the likelihood of sex. Overall, we found that men were better at predicting when they would not have sex than when they would, particularly if any likelihood value greater than $0 \%$ was treated as indicative that sex might occur. We found no evidence that men's accuracy of prediction was affected by whether it was a weekend or whether they were using substances, though both did increase the probability of sex.

Discussion: These results suggested that, were men taking event-driven intermittent PrEP, 14\% of doses could have been safely skipped with a minimal rate of false negatives using guidelines of taking a dose unless there was no chance (i.e., $0 \%$ likelihood) of sex on the following day. This would result in a savings of over $\$ 1,300$ per year in medication costs per participant.

Keywords: Gay and bisexual men; HIV/AIDS; intermittent PrEP; event-driven PrEP; predicting sexual behavior 


\section{Introduction}

Gay, bisexual, and other men who have sex with men (GBMSM) continue to disproportionately bear the burden of the epidemic. ${ }^{1,2}$ There is an urgent need for effective biomedical and behavioral strategies to reduce incidence in this population. In 2012, the FDA approved the use of once-daily Truvada (Emtricitabine/Tenofovir) for use among HIV-negative individuals as HIV Pre-Exposure Prophalyxis (PrEP), and the CDC subsequently recommended combined once-daily Truvada to be coupled with traditional HIV prevention/education (e.g., condom use). ${ }^{3-5}$ PrEP is, at present, one of the most promising biomedical prevention tools for GBMSM in the US.

Daily dosing may not be necessary for all PrEP candidates. First, most individuals are not exposed to HIV on a daily basis and Truvada has a relatively long half-life in plasma and peripheral blood mononuclear cells ( $\geq 60$ and 39 hours). ${ }^{6,7}$ This suggests less frequent dosing might maintain sufficient levels to prevent infection in the event of an exposure. In fact, results from the iPrEx Open Label Extension (OLE) study—a PrEP demonstration project—found no new HIV infections among those who took Truvada at least 4 days per week. ${ }^{8}$ Second, daily dosing, compared with less frequent dosing, might be considered burdensome in several ways, including the physical act of swallowing the pill, the cognitive burden of remembering daily, the potential for increased treatment side effects or toxicity, and the financial costs. ${ }^{9-11}$

As data from animal models regarding the efficacy of intermittent PrEP are promising, ${ }^{12,13}$ two intermittent PrEP dosing strategies are being studied in humans as alternatives to daily dosing-time-driven and event-driven dosing. In the HPTN 067 (ADAPT) study, daily dosing is being compared to both time-driven and event-driven dosing. The timedriven arm involves one dose of Truvada taken twice weekly regardless of sexual activity and an 
additional post-exposure dose after any sexual act; the event-driven arm in the ADAPT trial involves one dose 24-48 hours before sexual intercourse and a second within two hours of intercourse. ${ }^{14}$ In the IPERGAY trial, ${ }^{15}$ a different form of event-driven dosing is utilized-two doses of Truvada are taken 2-24 hours before any anticipated sexual intercourse and, if sexual behavior occurs, two doses are taken with the first occurring 24 hours and a second 48 hours after sexual activity. On October 29, 2014, the IPERGAY study announced that preliminary results examined by their Data Safety Monitoring Board indicated a significant difference in HIV incidence between those receiving event-driven dosing and placebo, and closed the placebo arm. ${ }^{16}$ This suggests that event-based dosing may soon be among the recommended PrEP strategies, particularly since a recent study of potential PrEP users in Kenya found no differences in preferences for daily versus event-driven regimens. ${ }^{11}$ Although the full extent of the efficacy of intermittent dosing will not be known until these trials are completed, effectiveness of eventdriven dosing will likely depend on the ability of individuals to predict in advance when sex will occur.

There are important caveats to intermittent PrEP dosing strategies. Individuals who typically experience three or more exposures in a given week might be better served by daily PrEP. ${ }^{17}$ Similarly, event-driven PrEP requires individuals to dose shortly following the time that exposure occurs. In a study of GBMSM and female sex workers, adherence rates for daily dosing $(83 \%)$ were significantly higher than for intermittent dosing (55\%) and dosing after sex (26\%), ${ }^{9}$ and similar findings emerged in a study of HIV serodiscordant couples in Uganda. ${ }^{18}$ However, preliminary results from the IPERGAY trial suggest high adherence to intermittent dosing, with participants on average taking 15 doses per month. ${ }^{19}$ 
There is little research to suggest whether GBMSM can accurately predict when sexual events will occur. In a study of 1,013 U.S. men online, Volk et al. ${ }^{17}$ reported that $50.4 \%$ of GBMSM had no advanced planning prior to their last sexual encounter, and $8.2 \%$ reported planning only minutes in advance. The remaining men reported planning hours $(22.4 \%), 1-3$ days $(10.7 \%)$, or more than 3 days in advance $(8.4 \%)$. Similarly, in a study of 823 GBMSM in Bangkok, Thailand, men reported that more than two-thirds of their sex events from the previous week were planned, though how far in advance men planned the events was not reported. ${ }^{20}$ Both studies suggested some men were able to plan sexual events in advance. However, these analyses relied upon retrospective recall data potentially overestimating the extent to which individuals were able to predict their own behavior.

Addressing these limitations, the present study was designed to use prospective (i.e., daily diary) analyses to examine the accuracy of GBMSM in predicting the next day's sexual behavior for the purposes of informing future research on alternative PrEP dosing schedules. Our sample included GBMSM who reported high numbers of sexual partners-a group explicitly targeted in CDC guidelines for PrEP utilization. ${ }^{21}$ Our a priori assumption is that the most important classification statistic to minimize is false negatives-where the individual believes they will not have sex the next day and does not take a PrEP dose, but ultimately has sex on the next day. A secondary classification priority was avoiding medication waste by taking it unnecessarily the day before sex does not occur (i.e., precision).

\section{Method}

Analyses were conducted on data from The Pillow Talk Project, a study of highly sexually active (i.e., $\geq 9$ male partners in 90 days) gay and bisexual men (GBM) in New York City. ${ }^{22,23}$ The analyses were limited to the 208 men confirmed to be HIV-negative with a rapid 
HIV test. One man did not complete any of the daily diaries and was excluded from analyses. We did not implement the question that is the main focus of these analyses until July, 2012. As such, 115 men never received this question. This resulted in a sample of 92 men with an average completion rate of $78.1 \%(M=23.4$ days $)$ and median rate of $86.7 \%$ ( $M d n=26.0$ days $)$.

Analyses involved the creation of a time-lagged variable and as such, contiguous reporting was required (see "daily diary measures"). In all, 341 days' worth of data were unmatched, resulting in a final analytic dataset of 1,688 days across 92 HIV-negative, highly sexually active GBM.

\section{Participants and Procedures}

Potential participants completed a phone-based screening to assess eligibility, which was defined as: 1) at least 18 years of age; 2) biologically male and self-identified as male; 3) nine or more male sexual partners in the prior 90 days; ${ }^{24-28}$ 4) self-identification as gay, bisexual, or some other non-heterosexual identity; and 5) daily access to the Internet. Participants who met preliminary eligibility were emailed a link to an Internet-based computer-assisted self-interview (CASI), which included informed consent procedures. Men completed this one-hour survey at home followed by an in-person baseline appointment. Final eligibility and enrollment was confirmed during the in-person appointment, with sexual partner criteria confirmed using a timeline follow-back (TLFB) interview. Following the baseline, participants received a unique link to complete their diaries each night for 30 days. All procedures were approved by the Institutional Review Board of the City University of New York.

\section{Measures}

Online CASI measures. Participants were asked to report demographic characteristics, including sexual identity, age, race/ethnicity, education, and relationship status. 
Daily diary measures. The diary measure was based on previous studies conducted with GBM. ${ }^{29-31}$ Each day, participants reported on their substance use and we calculated one indicator ( $1=$ yes, $0=$ no) of whether the participant had consumed five or more alcoholic drinks (i.e., heavy drinking) and a second for whether they had used cocaine, crack, crystal meth, ecstasy, heroin, ketamine, or GHB (i.e., club drugs). Following those sections, participants were asked whether they had engaged in any sexual activity with another person and, if so, were asked a series of questions for each partner they reported for that day. For the purposes of these analyses, we recoded all partner-level data into a single day-level indicator of whether or not $(1=$ yes, $0=$ no) the participant had engaged in any anal sex with a casual male partner. At the end of the survey, participants were asked, "How likely is it that you will have anal sex with a casual male partner tomorrow?" and were given a visual analog-style sliding scale ranging from 0 (absolutely sure I will not) to 100 (absolutely sure I will). This variable was time-lagged such that it was matched with the sexual behavior report on the next day to estimate their accuracy in prediction. The variable was also centered at 50 and divided by 10 such that a one-unit increase corresponded to an increase of $10 \%$ in self-reported likelihood.

\section{Analytic Plan}

All models were conducted using multilevel logistic regression accounting for a firstorder autoregressive (i.e., AR1) structure in the repeated responses and a random intercept with an unstructured covariance matrix using SAS version 9.2's GLIMMIX procedure. To inform recommendations regarding cutoff points in likelihood ratings and their utility for when to take a PrEP dose prior to a sexual occasion, we estimated two theoretically relevant classification statistics across the range of self-reported likelihood of sex: the false negative rate (i.e., [below the cutoff but had sex]/[all those who had sex]; on what percentage of days when a pill would 
have been needed would the participant not have taken one at the specified cutoff?) and precision (i.e., [at or above the cutoff and had sex]/[all those at or above the cutoff]; on what percentage of days when a pill would have been taken was the dose actually needed?).

\section{Results}

As shown in Table 1, the sample was diverse with regards to race/ethnicity, employment status, and educational achievement, while a majority was gay-identified and single.

Additionally, nearly two-thirds of the sample engaged in sexual activity on three or more days per week. Figure 1 presents a histogram of the likelihood responses, with those greater than $0 \%$ collapsed into deciles. The most commonly reported single point on the likelihood scale was $0 \%$, with an average likelihood of 26.3\% (SD = 26.4\%; $\mathrm{Mdn}=19.0 \%$; IQR $=4-41 \%)$.

\section{[Table 1 Here]}

[Figure 1 Here]

We tested for linearity of the association between self-reported likelihood and behavior by creating 11 dichotomous indicators of each decile on the likelihood variable (e.g., 0\%, 1-10\%, 91-100\%) and examining their association with behavior. As can be seen in Figure 1, the association was primarily linear. We also explored this issue using both first-order and quadratic terms predicting the behavioral outcome and found no evidence of a significant quadratic effect. As such, further analyses were conducted examining a linear association between likelihood reports and behavior. In a model with only the continuous likelihood rating, the odds of sex on an average day with a $50 \%$ likelihood rating was 0.30 (probability $=0.23$ ) and this increased by $36 \%(\mathrm{OR}=1.36)$ for every $10 \%$ increase in self-reported likelihood. We found that the effect of time (i.e., the day of diary completion) did not have an influence on the outcome, but that weekend days (i.e., Friday, Saturday, Sunday) versus weekdays were associated with increased 
odds of anal sex with casual partners $(\mathrm{OR}=1.31,95 \% \mathrm{CI}[1.00,1.72])$. We found no evidence of an interaction between likelihood ratings and whether or not it was a weekend day. That is, though men tended to be more sexually active on the weekends, this neither improved nor worsened their accuracy of prediction. As a result of its significant main effect, we subsequently adjusted all models for whether or not the day on which the behavior was measured on a weekend day.

In the next series of multilevel models, we separately tested level 1 (i.e., daily) substance use variables for main effects on the outcome as well as interaction effects with the likelihood rating to examine whether certain contextual effects might influence the accuracy of participants' reports. Both heavy drinking $(\mathrm{AOR}=1.63,95 \% \mathrm{CI}[1.06,2.49])$ and drug use $(\mathrm{AOR}=2.64$, 95\%CI[1.21, 5.75]) on a given day had significant main effects on whether or not participants engaged in casual anal sex, but neither significantly interacted with likelihood ratings to decrease participants' accuracy of prediction $(\mathrm{AOR}=0.97,95 \% \mathrm{CI}[0.84,1.13]$; AOR=0.83, 95\% CI $[0.66$, 1.05], respectively).

[Figure 2 Here]

Figure 2 provides estimates of classification statistics across the observed range of selfreported likelihoods of having sex on the next day. As shown, the rate of false negatives increased sharply as participants increased their ratings of sex likelihood. If a threshold even as low as $10 \%$ sex likelihood was set for taking a PrEP dose, then a missed dose would have occurred on $12.6 \%$ of days when an individual would have needed a dose prior to sex. At this same threshold of $10 \%$ likelihood, only $20.5 \%$ of the doses that would be taken would actually have been subsequently needed (i.e., $79.5 \%$ of the time they would be taking a dose that was unnecessary because sex ultimately did not occur the next day). Using anything greater than $0 \%$ 
likelihood of having sex as the cutoff, the rate of false negatives was $3.8 \%$ and precision was similar to values at $10 \%$ likelihood (17.3\% versus $20.5 \%$ ). Ratings of $0 \%$ likelihood occurred on $14.0 \%$ of days (i.e., ratings greater than $0 \%$ likelihood occurred on $86 \%$ of days). Overall, there were no levels of self-reported likelihood where participants had good precision in their predictions; even at a cutoff of $100 \%$, doses would be unnecessary $41.4 \%$ of the time that they were taken.

\section{Discussion}

Based on our results, there appear to be two approaches that could be used to inform event-driven intermittent PrEP use among GBMSM where a dose needs to be taken a day prior to sex. The first strategy would counsel individuals to "Take a dose today unless you are not going to have sex tomorrow." Under this strategy, classification statistics suggest a recommended a $1 \%$ or greater chance of sex the next day be used; in other words, individuals would be instructed to only skip a daily dose when they were certain there would be no chance of having sex the next day. In this sample, the $0 \%$ likelihood value produced a rate of $3.8 \%$ false negatives (i.e., cases where sex was not predicted but did occur the next day). Although a $0 \%$ chance of sex may seem like a very stringent criterion, it has a number of beneficial properties beyond the important characteristic of minimizing false negatives. This value was the most common prediction point—even in this sample of highly sexually active men—with participants reporting $0 \%$ likelihood an average of 4.2 days per month. In our sample, this means that $14 \%$ of doses could have been skipped without increasing risk for transmission in the proceeding 24 hours. At an estimated cost of $\$ 25.86$ per dose, ${ }^{32}$ this would save an average of $\$ 1,321.45$ per year in medication costs per participant (i.e., more than 1.5 months' worth of medication). Given known innumeracy issues in individuals' ability to interpret probabilities, ${ }^{33}$ and the fact that men 
were better at predicting when they would not have sex than when they would, another beneficial feature of this cut point is that it is easy to apply (i.e., "only skip a dose if you are certain there is no chance you will have sex tomorrow") versus an intermediate value. Perhaps most importantly, the consequence of making a wrong decision under this strategy are rather benign (i.e., an unnecessary dose is taken).

A second potential counseling strategy for those on event-driven dosing would be to focus on sexual forecasting, working with GBMSM to identify which days of the week they are likely to have sex and instruct them to "Take a dose if there is any chance you are going to have sex the next day." This is similar to the counseling approach being used in HPTN 067 (ADAPT) and will be effective when users can have high precision in their predictions at the same time as avoiding false negatives. However, our study suggests that, among highly sexually active GBM, this approach would not be effective because classification statistics indicate the men had low levels of precision in predicting their sexual behavior across the full range of likelihood ratings $(15.5 \%-58.6 \%)$. Even at a reported $100 \%$ likelihood of sex occurring the next day, precision was only $58.6 \%$ and the false negative rate was unacceptably high at this likelihood level (i.e., 94\%). Most importantly, the consequences of making a wrong decision under this strategy are high (i.e., a necessary dose would not be taken, therefore increasing risk of infection).

\section{[Table 2 Here]}

Importantly, our findings also suggest that the fixed dosing schedule may be the most appropriate for men who have at least one sexual event per week. Table 2 contains a hypothetical week for the ADAPT and IPERGAY trial dosing schedules based on sexual behavior. As can be seen, the fixed dosing schedule in ADAPT would lead to a greater number of doses than the other two schedules only during weeks when no sexual activity is anticipated or occurs (within 
the table, perfect correspondence between prediction and behavior is assumed). In contrast, the IPERGAY event-driven dosing schedule may lead to as many as eight doses in a week with only two anticipated and actual sex events, which is more than the number of doses taken with daily PrEP. The event-driven dosing in ADAPT and IPERGAY is contingent upon successfully predicting one's sexual behavior such that a pre-coital dose can be taken-in ADAPT's eventdriven dosing, a single dose is taken between $24-48$ hours prior to sexual activity while IPERGAY's event-driven schedule calls for two doses taken 2-24 hours prior to sexual activity. Table 2 assumes perfect correspondence between anticipated and actual sexual behavior, which the current study demonstrated is rarely the case. Rather, our findings demonstrate that, in general, attempting to make a prediction approximately 24 hours in advance led to very imprecise estimates and would ultimately lead to pre-coital doses being taken on all but approximately one day per week, on average. In contrast to this, the IPERGAY schedule, while requiring a greater number of pre-coital doses (i.e., 2 pills rather than one), could ultimately result in fewer doses per week if participants are more accurate at predicting sex within shorter intervals of time (i.e., the 2-24 hours recommended in the IPERGAY schedule). Although this is not depicted within the table due to the complexity, this may result in fewer pre-coital doses being taken due to greater accuracy in predicting sex at shorter intervals, though it would result in more doses being taken after actual engagement in sexual behavior.

Overall, it is impossible to say with the current data which dosing schedule will lead to the most adaptive plan due to the fact that all estimates were requested on the day prior rather than on the day of a sexual event as would be possible within the IPERGAY schedule. More information is required about the potential increases in accuracy at these shorter intervals to determine how many pills could ultimately be skipped safely and what the resultant cost savings 
would be. These data are not able to shed light on the potential efficacy of any of these dosing schedules. However, the data do suggest that men would meet the level of 4 doses per week with only one sexual event per week in IPERGAY and with two sexual events in the fixed dosing schedule of the ADAPT trial, though they may not receive a minimum of four doses at two sexual events per week using the event-driven dosing in ADAPT, depending on the distance between sexual events as can be seen in the final two hypothetical weeks in Table 2 . Given that recent research suggests that four doses may be optimal for preventing infection, ${ }^{8}$ and the recent discontinuation of the placebo arm in the IPERGAY trial suggesting efficacy of event-driven dosing, ${ }^{16}$ our data suggest that men who have one to two sexual events per week may be best advised by the IPERGAY event-driven dosing schedule given both its flexibility with pre-coital prediction intervals and the number of doses that would be achieved. However, for men for whom sexual behavior is more regular, the IPERGAY schedule may lead to more doses than would be required by daily PrEP and these men may be better suited to utilize the fixed ADAPT event-driven dosing schedule combined with the suggestion from these data that they take a precoital dose unless they believe there is no chance of sexual behavior on the following day. These results may lead to both the optimal dosing levels and cost savings.

In addition to informing strategies for how to counsel event-driven PrEP users on how to predict sex and adjust their dosing, this study also provided novel information on how well men could predict their future sexual behavior. In this sample of highly sexually active GBM, participants consistently overestimated the likelihood that sex would occur across the range of likelihoods. For example, when men predicted between $71-80 \%$ likelihood of having sex, the actual probability was $45.7 \%$. In the absence of data on such predictions among GBM who are less sexually active, it is unknown if this represents a general affirmative bias among GBM (or 
men in general), or whether the characteristics of this study population may moderate prediction tendency. For example, highly sexually active men may be more likely to predict sex will occur due to their higher overall frequency of sex. Other important findings included the lack of effect moderation due to weekend versus week day or substance use during sex; although both increased the probability of having sex, they did not decrease men's accuracy of prediction. This suggests that GBM may have accounted for these variables in their predictions of the likelihood of sex occurring.

This study is not without limitations. First, this was a sample of highly sexually active GBM in NYC, and therefore results may not generalize across the distribution of sexual activity levels. At the same time, the population is one of the primary targets for PrEP utilization, ${ }^{21}$ and therefore our findings have particular relevance. Second, it is important to note that the data came from an observational diary study and participants had no incentive to change their sexual behavior based on their predictions. In the case of event-driven PrEP, users may have an incentive to change their behavior depending on if they predicted sex and therefore took a PrEP dose on the prior day. These changes could include avoiding sex when a dose was not taken in advance, which would decrease false negatives, increasing condom use when a dose was not taken the prior day, or being more inclined to engage in sex on days after a dose was taken, which would increase the precision of their predictions. To the extent that this strategy of eventdriven intermittent PrEP dosing is effective at preventing HIV infection, then such an increase would be inconsequential. Lack of effectiveness, however, would produce negative consequences akin to risk compensation. ${ }^{34}$ Finally, the sample size was limited to fewer than 100 men, and thus we lacked power to examine many potential individual-level moderators of prediction accuracy, including level of risk. Moreover, the likelihood question, and thus the 
outcome examined, focused only on sex with casual partners. Future studies are needed that more carefully examine which individual characteristics make men better or worse at accurately predicting their behavior and incorporate the role of relationships within the analyses.

In conclusion, our unique daily diary data allowed for estimation of how well highly sexually active GBM can predict when sex will occur on the next day. Such data have been missing from the discussion of event-driven intermittent PrEP utilization. On an a priori basis we concluded that the most important classification statistic to minimize was false negatives, as they would serve to increase risk for infection because a needed dose it not taken. Our diary data indicated there were very few false negatives when participants indicated there was $0 \%$ chance of sex occurring the next day, and therefore suggest that the most effective approach for this population is to advise that a dose should be taken every day, unless the user is certain that sex will not occur the next day. This recommendation is essentially equivalent to daily PrEP minus days in which sex is not going to occur the next day. In the diary data there were many such days, and therefore this approach could produce considerable cost savings, which is critical because existing analyses indicate cost-effective implementation of daily PrEP would be challenging in the U.S. ${ }^{32,35,36}$. It is also expected that less frequent dosing would decrease sideeffects related to drug exposure, ${ }^{21}$ which could impact adherence. Given these benefits of eventdriven PrEP, future trials of this event-driven intermittent usage strategy are likely to be conducted if the results of current trials support efficacy. If our results are replicated in further studies, future event-driven PrEP trials may be able to increase efficacy by shifting their counseling strategy to one of "take a dose unless you are certain there is no chance sex will occur tomorrow." Such strategies may lead to even greater cost savings among men with less frequent sexual activity. 


\section{Acknowledgements}

This project was supported by a research grant from the National Institute of Mental Health (R01-MH087714; Jeffrey T. Parsons, Principal Investigator). H. Jonathon Rendina was supported in part by a National Institute of Mental Health Ruth L. Kirchstein Individual Predoctoral Fellowship (F31-MH095622). The content is solely the responsibility of the authors and does not necessarily represent the official views of the National Institutes of Health.

JTP, HJR, and BM planned and/or conducted statistical analyses. All authors were involved in the conception, design, and implementation of the project. All authors contributed to data interpretation, article writing and/or review, and editing. All authors reviewed a final version of the manuscript and approved it for submission.

The authors would like to acknowledge the contributions of the Pillow Talk Research Team, particularly John Pachankis, Ruben Jimenez, Demetria Cain, Sitaji Gurung, and Chris Cruz, as well as Arjee Restar and Raymond Moody who assisted with the review of the literature. We would also like to thank CHEST staff who played important roles in the implementation of the project: Chris Hietikko and Chloe Mirzayi, as well as our team of research assistants, recruiters, and interns. Finally, we thank Chris Ryan, Daniel Nardicio, and Stephan Adelson and the participants who volunteered their time for this study. 


\section{References}

1. CDC. Estimated HIV Incidence in the United States, 2007-2010. HIV Surveillance Supplemental Report. 2012;17(4).

2. CDC. CDC analysis provides new look at disproportionate impact of HIV and syphilis among U.S. gay and bisexual men. 2010; http://www.cdc.gov/nchhstp/Newsroom/msmpressrelease.html. Accessed Mar. 15, 2010.

3. Centers for Disease C, Prevention. Interim guidance: preexposure prophylaxis for the prevention of HIV infection in men who have sex with men. MMWR Morb. Mortal. Wkly. Rep. 2011;60(3):65-68.

4. Centers for Disease C, Prevention. Interim guidance for clinicians considering the use of preexposure prophylaxis for the prevention of HIV infection in heterosexually active adults. MMWR Morb. Mortal. Wkly. Rep. 2012;61(31):586-589.

5. Centers for Disease C, Prevention. Update to Interim Guidance for Preexposure Prophylaxis (PrEP) for the Prevention of HIV Infection: PrEP for injecting drug users. MMWR Morb. Mortal. Wkly. Rep. 2013;62(23):463-465.

6. Kearney BP, Flaherty JF, Shah J. Tenofovir disoproxil fumarate. Clin. Pharmacokinet. 2004;43(9):595-612.

7. Wang LH, Begley J, St. Claire III RL, Harris J, Wakeford C, Rousseau FS. Pharmacokinetic and pharmacodynamic characteristics of emtricitabine support its once daily dosing for the treatment of HIV infection. AIDS Res. Hum. Retroviruses. 2004;20(11):1173-1182. 
8. Grant RM, Anderson PL, McMahan V, et al. Uptake of pre-exposure prophylaxis, sexual practices, and HIV incidence in men and transgender women who have sex with men: A cohort study. The Lancet Infectious Diseases. 2014.

9. Mutua G, Sanders E, Mugo P, et al. Safety and adherence to intermittent pre-exposure prophylaxis (PrEP) for HIV-1 in African men who have sex with men and female sex workers. PLoS One. 2012;7(4):e33103.

10. Brooks RA, Kaplan RL, Lieber E, Landovitz RJ, Lee SJ, Leibowitz AA. Motivators, concerns, and barriers to adoption of preexposure prophylaxis for HIV prevention among gay and bisexual men in HIV-serodiscordant male relationships. AIDS Care. 2011:1-10.

11. Roberts ST, Heffron R, Ngure K, et al. Preferences for daily or intermittent pre-exposure prophylaxis regimens and ability to anticipate sex among HIV uninfected members of Kenyan HIV serodiscordant couples. AIDS Behav. 2014.

12. Cong ME, Youngpairoj AS, Zheng Q, et al. Protection against rectal transmission of an emtricitabine-resistant simian/human immunodeficiency virus SHIV162p3M184V mutant by intermittent prophylaxis with Truvada. J. Virol. 2011;85(15):7933-7936.

13. Garcia-Lerma JG, Otten RA, Qari SH, et al. Prevention of rectal SHIV transmission in macaques by daily or intermittent prophylaxis with emtricitabine and tenofovir. PLoS Med. 2008;5(2):e28.

14. Curran K, Mugo NR, Kurth A, et al. Daily Short Message Service Surveys to Measure Sexual Behavior and Pre-exposure Prophylaxis Use Among Kenyan Men and Women. AIDS Behav. 2013;17(9):2977-2985.

15. AVAC. IPERGAY. 2014; http://www.avac.org/trial/ipergay. Accessed Jun 10, 2014. 
16. ANRS France REcherche Nord \& Sud Sida-HIV Hépatites. A significant breakthrough in the fight against HIV/AIDS: A drug taken at the time of sexual intercourse effectively reduces the risk of infection [press release]. Paris, France, October 29, 2014. Available at: http://www.anrs.fr/content/download/6008/32756/file/Press\%20release\%20IPERGAYWEB.pdf.

17. Volk JE, Liu A, Vittinghoff E, et al. Sexual frequency and planning among at-risk men who have sex with men in the United States: implications for event-based intermittent pre-exposure prophylaxis. J. Acquir. Immune Defic. Syndr. 2012;61(1):112-115.

18. Kibengo FM, Ruzagira E, Katende D, et al. Safety, Adherence and Acceptability of Intermittent Tenofovir/Emtricitabine as HIV Pre-Exposure Prophylaxis (PrEP) among HIV-Uninfected Ugandan Volunteers Living in HIV-Serodiscordant Relationships: A Randomized, Clinical Trial. PLoS One. 2013;8(9):e74314.

19. Fonsart J, Capitant C, Spire B, et al. High adherence rate to intermittent oral PrEP with TDF/FTC among high risk MSM (ANRS Ipergay). Paper presented at: 20th International AIDS Conference2014; Melbourne, Australia.

20. van Griensven F, Thienkrua W, Sukwicha W, et al. Sex frequency and sex planning among men who have sex with men in Bangkok, Thailand: implications for pre- and post-exposure prophylaxis against HIV infection. J Int AIDS Soc. 2010;13:13.

21. US Public Health Service. Preexposure prophylaxis for the prevention of HIV infection in the United States - 2014: a clinical practice guideline. 2014.

22. Parsons JT, Rendina HJ, Ventuneac A, Cook KF, Grov C, Mustanski B. A psychometric investigation of the Hypersexual Disorder Screening Inventory among highly sexually 
active gay and bisexual men: An item response theory analysis. J. Sex. Med. 2013;10(12):3088-3101.

23. Pachankis JE, Rendina HJ, Ventuneac A, Grov C, Parsons JT. The Role of Maladaptive Cognitions in Hypersexuality Among Highly Sexually Active Gay and Bisexual Men. Arch. Sex. Behav. 2014;43(4):669-683.

24. Grov C, Parsons JT, Bimbi DS. Sexual compulsivity and sexual risk in gay and bisexual men. Arch. Sex. Behav. 2010;39(4):940-949.

25. Parsons JT, Bimbi DS, Halkitis PN. Sexual compulsivity among gay/bisexual male escorts who advertise on the Internet. Sexual Addiction \& Compulsivity. 2001;8:101-112.

26. Parsons JT, Kelly BC, Bimbi DS, DiMaria L, Wainberg ML, Morgenstern J. Explanations for the origins of sexual compulsivity among gay and bisexual men. Arch. Sex. Behav. 2008;37(5):817-826.

27. Stall R, Mills TC, Williamson J, Hart T, Greenwood G, Paul J, et al. Association of cooccurring psychosocial health problems and increased vulnerability to HIV/AIDS among urban men who have sex with men. Am. J. Public Health. 2003;93(6):939-942.

28. Stall R, Paul JP, Greenwood G, et al. Alcohol use, drug use and alcohol-related problems among men who have sex with men: the Urban Men's Health Study. Addiction. 2001;96(11):1589-1601.

29. Grov C, Golub SA, Mustanski B, Parsons JT. Sexual compulsivity, state affect, and sexual risk behavior in a daily diary study of gay and bisexual men. Psychol. Addict. Behav. 2010;24(3):487.

30. Mustanski B. Are sexual partners met online associated with HIV/STI risk behaviours? Retrospective and daily diary data in conflict. AIDS Care. 2007;19(6):822-827. 
31. Mustanski B. The influence of state and trait affect on HIV risk behaviors: A daily diary study of MSM. Health Psychol. 2007;26(5):618.

32. Juusola JL, Brandeau ML, Owens DK, Bendavid E. The cost-effectiveness of preexposure prophylaxis for HIV prevention in the United States in men who have sex with men. Ann. Intern. Med. 2012;156(8):541-550.

33. Konold C, Pollatsek A, Well A, Lohmeier J, Lipson A. Inconsistencies in students' reasoning about probability. Journal for Research in Mathematics Education. $1993 ; 24: 392-392$.

34. Golub SA, Kowalczyk W, Weinberger CL, Parsons JT. Preexposure prophylaxis and predicted condom use among high-risk men who have sex with men. J. Acquir. Immune Defic. Syndr. 2010;54(5):548-555.

35. Paltiel AD, Freedberg KA, Scott CA, et al. HIV preexposure prophylaxis in the United States: impact on lifetime infection risk, clinical outcomes, and cost-effectiveness. Clin. Infect. Dis. 2009;48(6):806-815.

36. Gomez GB, Borquez A, Case KK, Wheelock A, Vassall A, Hankins C. The cost and impact of scaling up pre-exposure prophylaxis for HIV prevention: a systematic review of cost-effectiveness modelling studies. PLoS Med. 2013;10(3):e1001401. 
[See PowerPoint Slide 1]

Figure 1. This figure displays a histogram of the frequency of self-reported likelihood within the bar chart while also displaying the model-implied probability of anal sex with a casual partner based on 11 percentiles of self-reported likelihood as a solid black line (the $95 \%$ confidence interval for the predicted values is displayed as a gray shadow around the solid line). The dotted black line serves as a referent for what a line of perfect correspondence between reported likelihood and actual behavior. As can be seen, participants tended towards lower predictions regarding the likelihood of sex on the next day, and the association between self-reported likelihood and subsequent engagement in sex was relatively linear. 
[See PowerPoint Slide 2]

Figure 2. Classification statistics for predicting anal sex with a casual male partner across the range of self-reported likelihood of having sex the next day. The false negative rate represents the proportion of false negatives (i.e., those who had sex but were not predicted to based on their likelihood score) over all actual positives (i.e., people who had sex). Precision represents the rate of true positives (i.e., people who were predicted to have sex based on their likelihood scores and actually did) over all predicted positives (i.e., people who were predicted to have sex based on their likelihood score. 
Table 1.

Demographic characteristics of the sample.

\begin{tabular}{|c|c|c|}
\hline Variable & $n$ & $\%$ \\
\hline \multicolumn{3}{|l|}{ Race/Ethnicity } \\
\hline Black & 13 & 14.1 \\
\hline Latino & 13 & 14.1 \\
\hline White & 50 & 54.3 \\
\hline Multiracial/Other & 16 & 17.4 \\
\hline \multicolumn{3}{|l|}{ Sexual Orientation } \\
\hline Gay, queer, or homosexual & 77 & 83.7 \\
\hline Bisexual & 15 & 16.3 \\
\hline \multicolumn{3}{|l|}{ Employment Status } \\
\hline Full-time & 35 & 38 \\
\hline Part-time & 29 & 31.5 \\
\hline Student (unemployed) & 9 & 9.8 \\
\hline Unemployed & 19 & 20.7 \\
\hline \multicolumn{3}{|l|}{ Highest Educational Attainment } \\
\hline High school diploma or GED & 6 & 6.5 \\
\hline Some college or Associate's degree & 24 & 26.1 \\
\hline Bachelor's or other 4-year degree & 40 & 43.5 \\
\hline Graduate degree & 22 & 23.9 \\
\hline \multicolumn{3}{|l|}{ Relationship Status } \\
\hline Single & 77 & 83.7 \\
\hline Partnered & 15 & 16.3 \\
\hline \multicolumn{3}{|l|}{ Days of Sex Per Week } \\
\hline Fewer than 3 & 33 & 35.9 \\
\hline \multirow[t]{2}{*}{3 or More } & 59 & 64.1 \\
\hline & $M$ & $S D$ \\
\hline Age & 33.5 & 11.5 \\
\hline
\end{tabular}

Note: $N=92$. 
Table 2.

Hypothetical dosing based on sexual activity in a given week for the ADAPT and IPERGAY dosing schedules.

\begin{tabular}{lccccccccc} 
& Mon & Tues & Weds & Thurs & Fri & Sat & Sun & TOTAL \\
& Sex Event? & No & No & No & No & No & No & No & 0 Days \\
\hline ADAPT - Fixed & 1 Fixed & -- & -- & 1 Fixed & -- & -- & -- & 2 Doses \\
ADAPT - Event-Driven & -- & -- & -- & -- & -- & -- & -- & 0 Doses \\
IPERGAY - Event-Driven & -- & -- & -- & -- & -- & -- & -- & 0 Doses
\end{tabular}

\begin{tabular}{|c|c|c|c|c|c|c|c|c|}
\hline Sex Event? & No & Yes & No & No & No & No & No & 1 Day \\
\hline ADAPT - Fixed & 1 Fixed & 1 Boost & -- & 1 Fixed & -- & -- & -- & 3 Doses \\
\hline ADAPT - Event-Driven & 1 Pre & 1 Post & -- & -- & -- & -- & -- & 2 Doses \\
\hline IPERGAY - Event-Driven & -- & 2 Pre & 1 Post & 1 Post & -- & -- & -- & 4 Doses \\
\hline
\end{tabular}

\begin{tabular}{|c|c|c|c|c|c|c|c|c|}
\hline Sex Event? & No & Yes & Yes & No & No & No & No & 2 Days \\
\hline ADAPT - Fixed & 1 Fixed & 1 Boost & 1 Boost & 1 Fixed & -- & -- & -- & 4 Doses \\
\hline ADAPT - Event-Driven & 1 Pre & 1 Post & 1 Post & & -- & -- & -- & 3 Doses \\
\hline IPERGAY - Event-Driven & -- & 2 Pre & 1 Post & & 1 Post & -- & -- & 5 Doses \\
\hline Sex Event? & No & Yes & No & No & Yes & No & No & 2 Days \\
\hline ADAPT - Fixed & 1 Fixed & 1 Boost & -- & 1 Fixed & 1 Boost & -- & -- & 4 Doses \\
\hline ADAPT - Event-Driven & 1 Pre & 1 Post & -- & 1 Pre & 1 Post & -- & -- & 4 Doses \\
\hline IPERGAY - Event-Driven & -- & 2 Pre & 1 Post & 1 Post & 2 Pre & 1 Post & 1 Post & 8 Doses \\
\hline
\end{tabular}




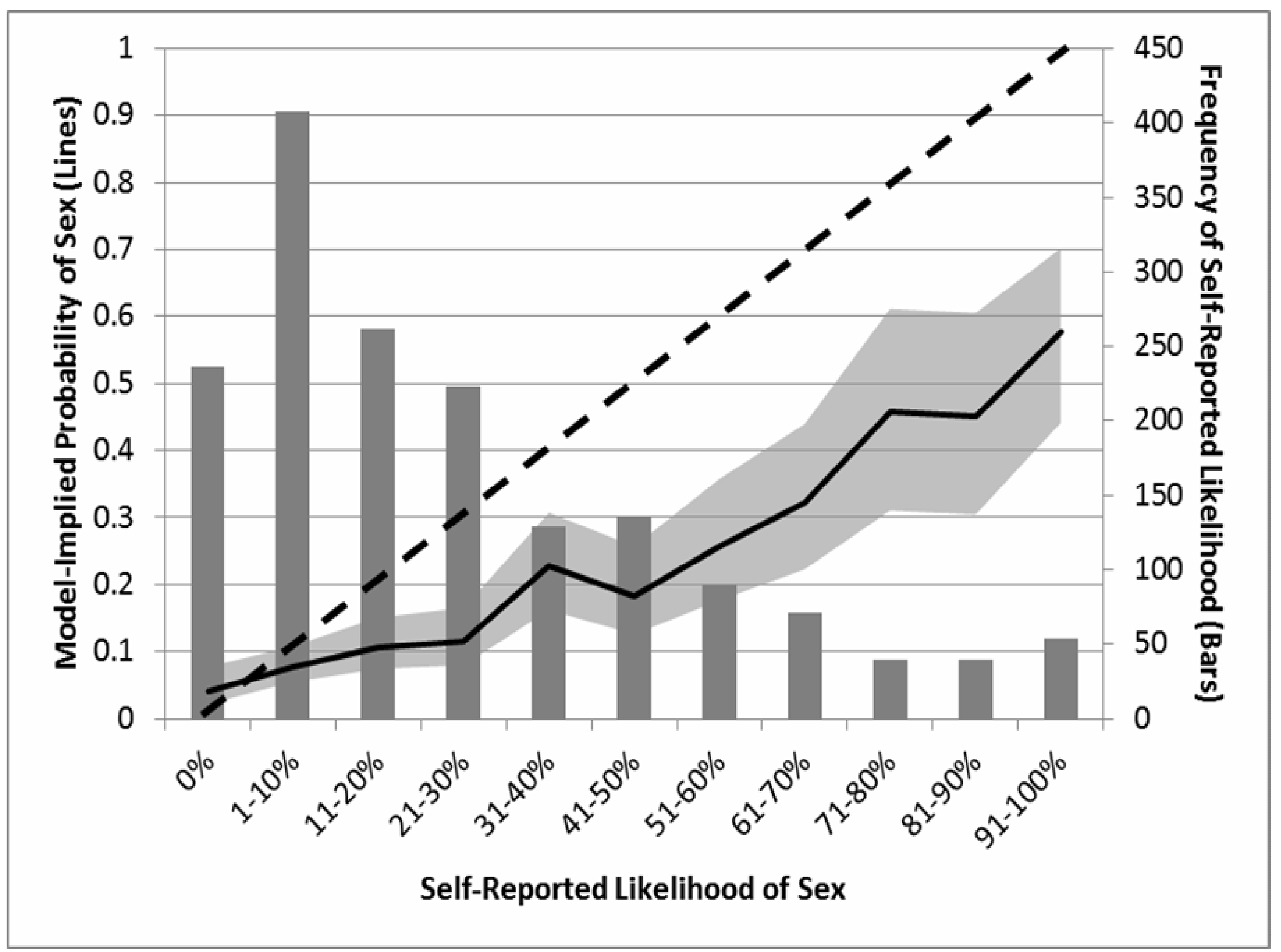

Copyright (C) 2015 Wolters Kluwer Health, Inc. Unauthorized reproduction of this article is prohibited. 


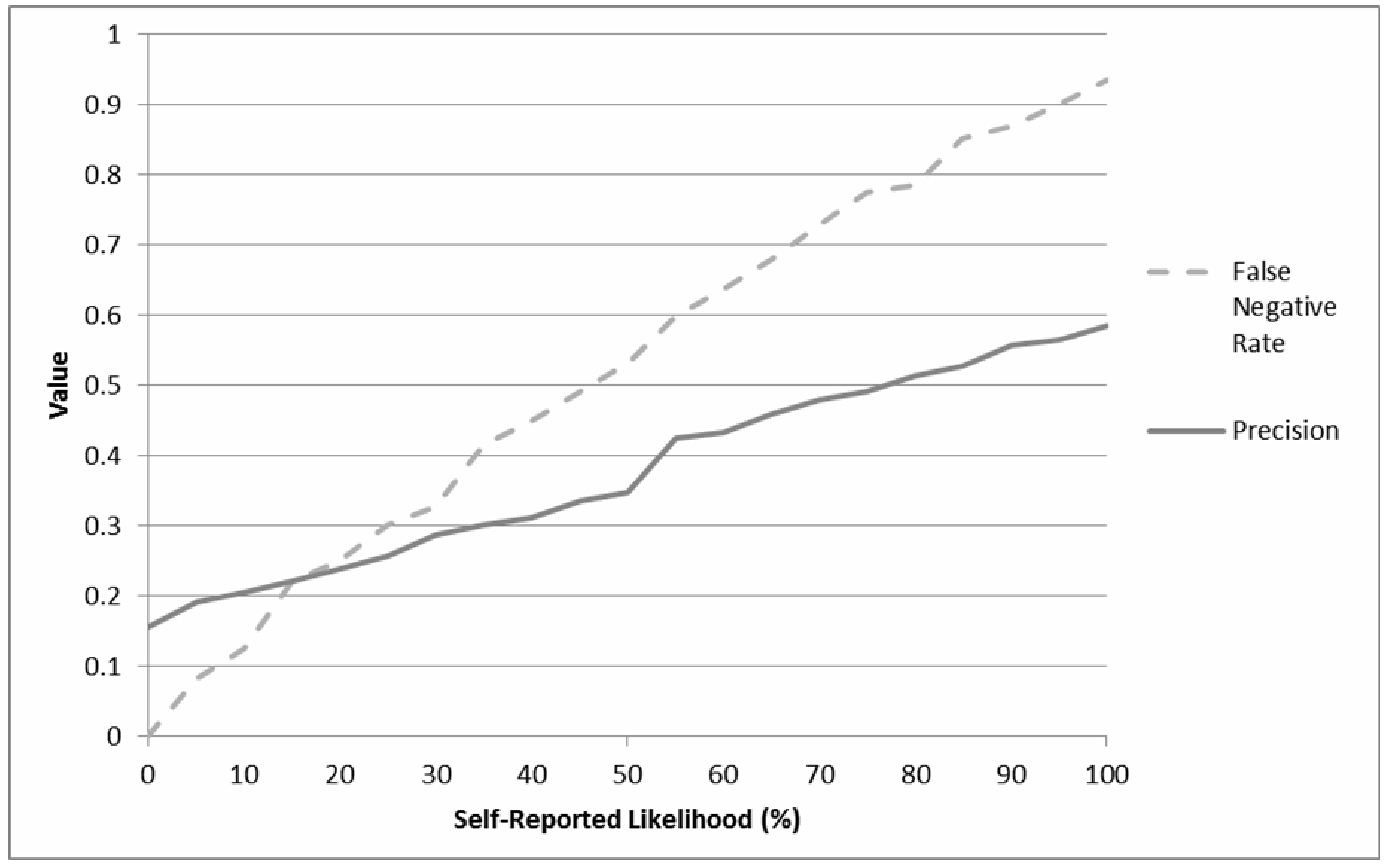

Copyright $(02015$ Wolters Kluwer Health, Inc. Unauthorized reproduction of this article is prohibited. 